\title{
Individuelle Begleitprogramme für Psoriatiker
}

In Österreich leben rund 160.000 Menschen mit Psoriasis. Wie gut Betroffene mit der Erkrankung umgehen können, ist neben dem Schweregrad auch sehr stark vom selbstverantwortlichen Erkrankungsmanagement abhängig (• Abb. 1). Dafür gibt es auch digitale Unterstützung: Die MyPso-App steht zusätzlich zu Quality$\mathrm{Care}^{\mathrm{TM}}$, einem umfassenden Service- und Begleitprogramm für Psoriatiker und deren Angehörige, kostenlos zum Download zur Verfügung.

\section{Sich online Hilfe zur Selbsthilfe holen: QualityCare ${ }^{\mathrm{Tm}}$}

QualityCare $^{\mathrm{TM}}$ ist ein unbürokratisches Serviceprogramm für Menschen mit Schuppenflechte und deren Angehörige. Auf der Online-Plattform www.qualitycare.co.at werden nach Anmeldung wichtige Informationen rund um die Erkrankung zur Verfügung gestellt - und zwar für jeden Nutzer individuell auf seiner „persönlichen Seite“. Diese Informationen werden auf Wunsch zusätzlich per E-Mail oder SMS versandt. Das kostenlose und vertrauliche Serviceangebot unterstützt alle Psoriatiker und kann auch von Ärzten im Sinne einer umfassenden Patientenbetreuung genützt werden.

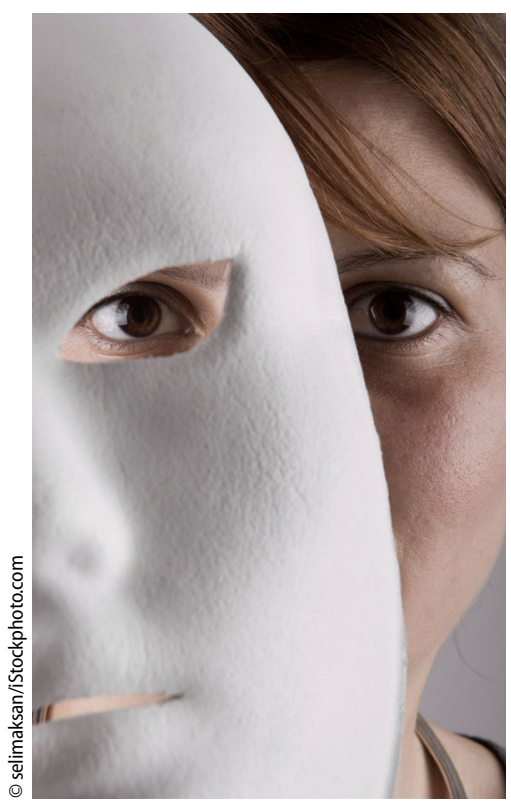

Abb. $1 \Delta$ Mit MyPso sind Psoriatiker nicht mehr allein. Sie können den eigenen Krankheitsverlauf dokumentieren und sehen, wie es anderen geht

\section{Die MyPso-App begleitet durch den Alltag}

Die MyPso-App (s. Kasten) liefert einen genauen Überblick über die Entwicklung der Erkrankung. Die App kann alle individuellen Psoriasis-Symptome und Auslöser aufzeichnen und Trends und Entwicklungen im Krankheitsverlauf feststellen (• Abb. 2). Daraus resultierende Erkenntnisse lassen sich zusammenfassen und herunterladen. Mit einfachen Grafiken werden die Muster der Schübe und Symptome veranschaulicht. Zudem können mit Hilfe der App Berichte erstellt werden, die auch dem Arzt einen wertvollen Einblick in den Krankheitsverlauf zwischen den Arztterminen liefern und dabei helfen können, die Therapie bei Bedarf leichter anzupassen.

\section{Anonymisierte Vergleichsdaten}

Geteiltes Leid ist bei Schuppenflechte nicht unbedingt halbes Leid. Aber zu sehen, dass andere Menschen

\section{Die MyPso App ...}

- veranschaulicht den Therapie-Fortschritt

- hilft, Auslöser von Schüben zu erkennen

- vergleicht Erkenntnisse anonym mit denen anderer Psoriasis-Betroffener in der App, um Ähnlichkeiten und Unterschiede darzustellen

- erstellt einen Bericht, der als Unterstützung beim nächsten Arztbesuch dient

Erhältlich ist die App über den Apple App Store und Google play für Android.

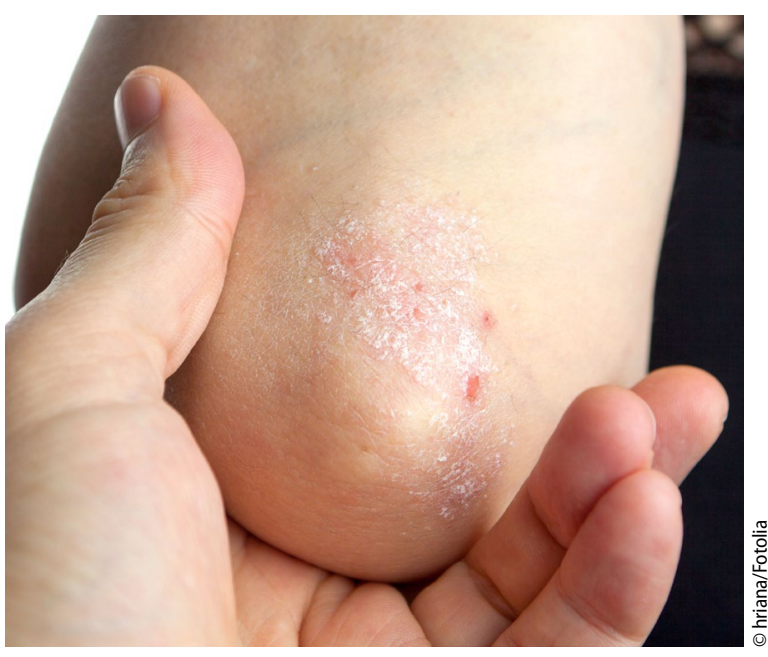

Abb. 2 \ Die MyPso-App kann alle individuellen Psoriasis-Symptome und Auslöser aufzeichnen und Trends und Entwicklungen im Krankheitsverlauf dokumentieren

mit Schuppenflechte auch nicht immer gleich gut mit ihrer Erkrankung zurechtkommen, kann helfen, sich selbst nicht zu sehr unter Druck zu setzen. Damit Patienten verfolgen können, wie es anderen gerade geht, zeigt MyPso die anonymisierten Daten der anderen Nutzer. Nicht zuletzt gibt die MyPso App praktische Tipps für den Alltag mit Psoriasis. So gelingt es leichter, schrittweise den Umgang mit der Erkrankung und in der Folge auch die Lebensqualität spürbar zu verbessern.

hautnah $2016 \cdot 15: 13$

DOI 10.1007/s12326-016-0182-8

Online publiziert: 27. Januar 2016 C) Springer-Verlag Wien 2016
Quelle: Presseaussendung eXakt PR 